\title{
CONTINUITY OF BEST APPROXIMANTS
}

\author{
D. LANDERS AND L. ROGGE
}

\begin{abstract}
Let $C_{n}, n \in N$, be $\Phi$-closed lattices in an Orlicz-space $L_{\phi}(\Omega, \mathcal{Q}, \mu)$ and assume that $C_{n}$ increases or decreases to a $\Phi$-closed lattice $C_{\infty}$. Let $f_{n}, n \in \mathrm{N}$, be $Q$-measurable real valued functions with $f_{n} \rightarrow f \mu$-a.e. and sup $\left|f_{n}\right| \in L_{\Phi}$. If $g_{n}$ is a best $\Phi$-approximant of $f_{n}$ in $C_{n}$ it is shown that $\lim _{n \in N} g_{n}$ and $\varlimsup_{n \in N} g_{n}$ are best $\Phi$-approximants of $f$ in $C_{\infty}$.
\end{abstract}

1. Introduction and notations. Let $(\Omega, \mathcal{Q}, \mu)$ be a measure space and $\Phi: \mathbf{R}_{+} \rightarrow \mathbf{R}_{+}$ be a convex function with $\Phi(0)=0$ and $\Phi \neq 0$. Denote by $L_{\Phi}(\Omega, \mathcal{Q}, \mu)$ respectively $L_{\Phi}^{\infty}(\Omega, \mathcal{Q}, \mu)$ the system of all $\mu$-equivalence classes of $\mathcal{Q}$-measurable functions $f$ such that $\int \Phi(\alpha|f|) d \mu<\infty$ for some $\alpha>0$ respectively for all $\alpha>0$. $L_{\Phi}$ and $L_{\Phi}^{\infty}$ are linear spaces with $L_{\Phi}^{\infty} \subset L_{\Phi}$; if $\Phi(x)=x^{P}$ then $L_{\Phi}=L_{\Phi}^{\infty}$ and we obtain the spaces $L_{p}, p \geqslant 1$. If $C \subset L_{\Phi}$ and $f \in L_{\Phi}$ denote by $\mu_{\Phi}(f \mid C)$ the system of all $g \in C$ fulfilling

$$
\int \Phi(|f-g|) d \mu=\inf _{h \in C} \int \Phi(|f-h|) d \mu .
$$

The elements of $\mu_{\Phi}(f \mid C)$ are called best $\Phi$-approximants of $f$, given $C$. The concept of best $\Phi$-approximants, given $C$, covers and unifies many important concepts of probability theory, e.g. the concepts in [1], [2], [6]; for more details see [4]. It is known that $\mu_{\Phi}(f \mid C) \neq \varnothing$ if $C$ is a lattice (i.e. $f, g \in C$ implies $f \wedge g, f \vee g \in C$ ) which is $\Phi$-closed (i.e. $f_{n} \in C, f \in L_{\Phi}$ and $f_{n} \uparrow f$ or $f_{n} \downarrow f$ imply $f \in C$ ); see Theorem 4 of [4]. In general, $\mu_{\Phi}(f \mid C)$ contains a lot of different elements; for instance if $\Phi(x)=x$ or if $C$ is not convex. This creates problems for proving limit theorems for best $\Phi$-approximants. In special cases-i.e. for $\Phi(x)=x^{p}, p>1$, and special types of $C$-limit results for best $\Phi$-approximants of $f$, given $C$, are easier to obtain for varying $f$ than for varying $C$; but in all these cases best approximants are unique. In the general context, however, the case of varying $f$ is more complex. There exist limit theorems for best $\Phi$-approximants of martingale type (see Theorem 21 and Theorem 22 of [4])-i.e. limit theorems for $g_{n} \in \mu_{\Phi}\left(f \mid C_{n}\right)$ with varying $C_{n}$-but there exist no continuity theorems for best $\Phi$-approximants-i.e. limit theorems for $g_{n} \in \mu_{\Phi}\left(f_{n} \mid C\right)$ with varying $f_{n}$. It is the aim of this paper to close this gap. We prove a limit theorem for best $\Phi$-approximants $g_{n} \in \mu_{\Phi}\left(f_{n} \mid C_{n}\right)$ where as well the functions $f_{n}$ as the $\Phi$-closed lattices $C_{n}$ may vary with $n \in \mathbf{N}$. We apply this result to obtain continuity of best approximants in the Orlicz-space norm of $L_{\Phi}$.

Received by the editors October 24, 1980.

1980 Mathematics Subject Classification. Primary 46E30; Secondary 41A50.

Key words and phrases. Best approximants, $\sigma$-lattices, conditional expectations, characterization. 
2. The results. Throughout the following let $(\Omega, \mathcal{Q}, \mu)$ be a measure space and $\Phi$ : $\mathbf{R}_{+} \rightarrow \mathbf{R}_{+}$be a convex function with $\Phi(0)=0$ and $\Phi \neq 0$. Then $\Phi$ is a continuous function with $\lim _{t>\infty} \Phi(t)=\infty$. If $C_{n} \subset L_{\Phi}, n \in \mathbf{N} \cup\{\infty\}$, we write $C_{n} \downarrow C_{\infty}$ if $C_{n} \supset C_{n+1}, n \in \mathrm{N}$, and $C_{\infty}=\bigcap_{n \in \mathrm{N}} C_{n}$. If $C_{n}$ are $\Phi$-closed lattices we write $C_{n} \uparrow C_{\infty}$ if $C_{n} \subset C_{n+1}$ and $C_{\infty}$ is the smallest $\Phi$-closed set containing $\cup_{n \in N} C_{n}$; then $C_{\infty}$ is a lattice, too (see [4, p. 229]).

1. Theorem. Assume that $L_{\Phi}=L_{\Phi}^{\infty}$. Let $C_{n} \subset L_{\Phi}, n \in \mathbf{N}$, be $\Phi$-closed lattices with $C_{n} \downarrow C_{\infty}$ or $C_{n} \uparrow C_{\infty}$ and $f_{n} \in L_{\Phi}, n \in \mathbf{N}$, with $f_{n} \rightarrow f \mu$-a.e. and $\sup _{n \in N}\left|f_{n}\right| \in L_{\Phi}$. Then for all $g_{n} \in \mu_{\Phi}\left(f_{n} \mid C_{n}\right), n \in \mathbf{N}$,

(i) $\underline{\lim }_{n \in \mathrm{N}} g_{n} \in \mu_{\Phi}\left(f \mid C_{\infty}\right), \varlimsup_{n \in \mathrm{N}} g_{n} \in \mu_{\Phi}\left(f \mid C_{\infty}\right)$.

(ii) $\sup _{n \in N}\left|g_{n}\right| \in L_{\Phi}$.

Proof. Let $C_{n} \downarrow C_{\infty}$. We prove that for each $g \in \mu_{\Phi}\left(f \mid C_{\infty}\right)$

$$
g \wedge \frac{\lim }{n \in \mathbf{N}} g_{n} \in \mu_{\Phi}\left(f \mid C_{\infty}\right), \quad g \vee \varlimsup_{n \in \mathbf{N}} g_{n} \in \mu_{\Phi}\left(f \mid C_{\infty}\right)
$$

As

$$
\frac{\lim }{n \in \mathbf{N}} g_{n}=\left(g \vee \varlimsup_{n \in \mathbf{N}} g_{n}\right) \wedge \frac{\lim }{n \in \mathbf{N}} g_{n}
$$

and

$$
\varlimsup_{n \in \mathrm{N}} g_{n}=\left(g \wedge \underset{n \in \mathrm{N}}{\lim } g_{n}\right) \vee \varlimsup_{n \in \mathrm{N}} g_{n},
$$

(1) implies (i).

Applying Lemma 3 to $C_{k} \supset \cdots \supset C_{n} \supset C_{\infty}$ we obtain for $n>k$

$$
\begin{aligned}
\int \Phi\left(\mid f_{k} \wedge \cdots\right. & \left.\wedge f_{n} \wedge f-g_{k} \wedge \cdots \wedge g_{n} \wedge g \mid\right) d \mu \\
& <\int \Phi\left(\left|f_{k} \wedge \cdots \wedge f_{n} \wedge f-g\right|\right) d \mu
\end{aligned}
$$

and

$$
\begin{aligned}
\int \Phi\left(\mid f_{k} \vee \cdots \vee\right. & \left.f_{n} \vee f-g_{k} \vee \cdots \vee g_{n} \vee g \mid\right) d \mu \\
& <\int \Phi\left(\left|f_{k} \vee \cdots \vee f_{n} \vee f-g\right|\right) d \mu
\end{aligned}
$$

From (2) we obtain for each $k$ with $n \rightarrow \infty$ according to the Lemma of Fatou that

$$
\begin{aligned}
\int \Phi\left(\left|f \wedge \bigwedge_{n>k} f_{n}-g \wedge \bigwedge_{n>k} g_{n}\right|\right) d \mu & \\
& <\frac{\lim }{n \in \mathbf{N}} \int \Phi\left(\left|f_{k} \wedge \cdots \wedge f_{n} \wedge f-g\right|\right) d \mu .
\end{aligned}
$$

Since $\sup _{n \in N}\left|f_{n}\right| \in L_{\Phi}$ by assumption, (4) implies by the Theorem of Lebesgue that for each $k \in \mathbf{N}$

$$
\int \Phi\left(\left|f \wedge \bigwedge_{n>k} f_{n}-g \wedge \bigwedge_{n>k} g_{n}\right|\right) d \mu \leqslant \int \Phi\left(\left|f \wedge \bigwedge_{n>k} f_{n}-g\right|\right) d \mu<\infty .
$$


As $f_{n} \rightarrow f$, $\sup _{n \in N}\left|f_{n}\right| \in L_{\Phi}$ we obtain from (5) with $k \rightarrow \infty$ using on the left side the Lemma of Fatou and on the right side the Theorem of Lebesgue that

$$
\int \Phi\left(\left|f-g \wedge \lim _{n \in \mathbf{N}} g_{n}\right|\right) d \mu<\int \Phi(|f-g|) d \mu<\infty .
$$

From (5) we obtain that $g \wedge \wedge_{n>k} g_{n} \in L_{\Phi}$. As $g \wedge \wedge_{n>k} g_{n}<\wedge_{n>k} g_{n}<g_{k}$ this implies

$$
\bigwedge_{n>k} g_{n} \in L_{\Phi}
$$

In the same way as (6) and (7) we obtain

$$
\int \Phi\left(\left|f-g \vee \varlimsup_{n \in \mathbf{N}} g_{n}\right|\right) d \mu<\int \Phi(|f-g|) d \mu<\infty
$$

and

$$
\bigvee_{n>k} g_{n} \in L_{\Phi}
$$

From (7) and (7)* applied to $k=1$ we obtain (ii). As $C_{n} \downarrow$ are $\Phi$-closed lattices we obtain with (7) that $\wedge_{n>j} g_{n} \in C_{k}$ for $j>k$. As $\lim _{n \in N} g_{n} \in L_{\Phi}$ by (ii), this implies $\underline{\lim }_{n \in N} g_{n} \in C_{k}$ for each $k \in N$ and hence $\underline{\lim }_{n \in N} g_{n} \in C_{\infty}$. Similarly $\varlimsup_{n \in \mathrm{N}} \bar{g}_{n} \in C_{\infty}$. Now (6), (6)* and $g \in C_{\infty}$ imply (1). This finishes the proof for the decreasing case.

Now let $C_{n} \uparrow C_{\infty}$. Applying Lemma 3 with $C_{n} \supset C_{n-1} \supset \cdots \supset C_{k}, k<n$, we obtain for $k<n$

$$
\int \Phi\left(\left|f_{k} \wedge \cdots \wedge f_{n}-g_{k} \wedge \cdots \wedge g_{n}\right|\right) d \mu<\int \Phi\left(\left|f_{k} \wedge \cdots \wedge f_{n}-g_{k}\right|\right) d \mu .
$$

Proceeding now as in the decreasing case, i.e. letting at first $n \rightarrow \infty$ and then $k \rightarrow \infty$ and using on the left sides the Lemma of Fatou and on the right sides the Theorem of Lebesgue we obtain

$$
\bigwedge_{n>k} g_{n} \in L_{\Phi}, \quad k \in \mathbf{N}
$$

and

$$
\int \Phi\left(\left|f-\lim _{n \in \mathbf{N}} g_{n}\right|\right) d \mu<\lim _{k \in \mathbf{N}} \int \Phi\left(\left|\bigwedge_{n>k} f_{n}-g_{k}\right|\right) d \mu .
$$

In the same way we obtain

$$
\bigvee_{n>k} g_{n} \in L_{\Phi}, \quad k \in \mathbf{N}
$$

and

$$
\int \Phi\left(\left|f-\varlimsup_{n \in \mathbf{N}} g_{n}\right|\right) d \mu<\lim _{k \in \mathbf{N}} \int \Phi\left(\left|\bigvee_{n>k} f_{n}-g_{k}\right|\right) d \mu<\infty .
$$

Relations (8) and ( $\left.8^{*}\right)$ directly imply

$$
\sup _{n \in N}\left|g_{n}\right| \in L_{\Phi} \quad \text { and } \quad \underset{n \in N}{\lim _{n}} g_{n}, \quad \varlimsup_{n \in \mathbb{N}} g_{n} \in C_{\infty} \text {. }
$$


Now apply Lemma 4 to $h_{k}=\left|f_{k}-g_{k}\right|$ and $r_{k}:=\left|\bigwedge_{n>k} f_{n}-f_{k}\right|$. Since $\sup _{k \in N}\left|g_{k}\right|$ $\in L_{\Phi}$ by (10), $\sup _{k \in N}\left|f_{k}\right| \in L_{\Phi}$ and $f_{k} \rightarrow f \mu$-a.e. by assumption we have $\sup _{k \in N} h_{k}$, $\sup _{k \in N} r_{k} \in L_{\Phi}$ and $r_{k} \rightarrow 0 \mu$-a.e., i.e. the assumptions of Lemma 4 are fulfilled. Hence we obtain

$$
\lim _{k \in \mathbf{N}} \int \Phi\left(h_{k}+r_{k}\right) d \mu=\frac{\lim }{k \in \mathbf{N}} \int \Phi\left(h_{k}\right) d \mu
$$

Since

$$
\Phi\left(\left|\bigwedge_{n>k} f_{n}-g_{k}\right|\right) \leqslant \Phi\left(\left|f_{k}-g_{k}\right|+\left|\bigwedge_{n>k} f_{n}-f_{k}\right|\right)=\Phi\left(h_{k}+r_{k}\right)
$$

(9) and (11) imply

$$
\int \Phi\left(\left|f-\frac{\lim }{n \in \mathbf{N}} g_{n}\right|\right) d \mu<\lim _{k \in \mathbf{N}} \int \Phi\left(\left|f_{k}-g_{k}\right|\right) d \mu .
$$

According to (12) and (10) we get $\lim _{n \in N} g_{n} \in \mu_{\Phi}\left(f \mid C_{\infty}\right)$ if we show that for all $g \in C_{\infty}$

$$
\frac{\lim }{k \in \mathbf{N}} \int \Phi\left(\left|f_{k}-g_{k}\right|\right) d \mu<\int \Phi(|f-g|) d \mu .
$$

Let $\hat{C}$ be the set of all $g \in L_{\Phi}$ fulfilling (13). Since $g_{k} \in \mu_{\Phi}\left(f_{k} \mid C_{k}\right), C_{k} \uparrow, f_{k} \rightarrow f$ $\mu$-a.e. and $\sup _{n \in \mathrm{N}}\left|f_{n}\right| \in L_{\Phi}$ it is easy to see that $\hat{C}$ is $\Phi$-closed with $\cup_{n \in \mathrm{N}} C_{n} \subset \hat{C}$. Hence $C_{\infty} \subset \hat{C}$, i.e. (13) holds for all $g \in \hat{C}$. Thus $\lim _{n \in \mathrm{N}} g_{n} \in \mu_{\Phi}\left(f \mid C_{\infty}\right)$ is shown; the proof for $\varlimsup_{n \in N} g_{n} \in \mu_{\Phi}\left(f \mid C_{\infty}\right)$ runs similarly (by using (9*) instead of (9)).

The martingale results, given in [4], hold for more general functions $\Phi$ than convex functions, namely for so-called $\mu$-functions. We do not know whether also the preceding theorem is true for this more general concept; the proof of Theorem 1 heavily uses the convexity of $\Phi$. Approximating $\lim _{n \in \mathrm{N}} g_{n} \mu$-a.e. by $g_{\tau_{n}}$ where $\tau_{n}$ is a sequence of finite stopping times for $g_{n}, n \in \mathrm{N}$, it can be seen that Theorem 1 is true for $\mu$-functions in the special case that $C_{n}$ is the system of $\mathbb{Q}_{n}$-measurable functions in $L_{\Phi}$, where $\mathbb{Q}_{n} \subset \mathbb{Q}$ are $\sigma$-fields, and $\mathbb{Q}_{n} \uparrow \mathbb{Q}_{\infty}$ or $\mathbb{Q}_{n} \downarrow \mathbb{Q}_{\infty}$. However, this procedure fails for arbitrary $\Phi$-closed lattices $C_{n}$.

If $f \in L_{\Phi}$ put $\|f\|_{\Phi}:=\inf \left\{a>0: \int \Phi(|f| / a) d \mu<1\right\}$. Then \|\|$_{\Phi}$ is a norm on $L_{\Phi}$ and the spaces $\left(L_{\Phi},\|\|_{\Phi}\right)$ are Banach-spaces; the well-known Orlicz spaces (see [5, p. 46]). If $C \subset L_{\Phi}$ and $f \in L_{\Phi}$ we denote by $\mu_{\|\|}(f \mid C)$ the set of all best \|\|$_{\Phi}$-approximants of $f$, given $C$, i.e. the set of all elements $g \in C$ with

$$
\|f-g\|_{\Phi}=\inf \left\{\|f-h\|_{\Phi}: h \in C\right\} .
$$

The concept of best \|\|$_{\Phi}$-approximants and its connection with the concept of best $\Phi$-approximants has been investigated in [4]. If $\Phi$ is strictly convex and if $L_{\Phi}=$ $L_{\Phi}^{\infty}$, then for each $\Phi$-closed convex lattice $C \subset L_{\Phi}$ and each $f \in L_{\Phi}$ there exist a unique best $\Phi$-approximant and a unique best \|\|$_{\Phi}$-approximant of $f$, given $C$ (see Corollary 5 and Corollary 13 of [4]), we denote these unique elements by $\mu_{\Phi}(f \mid C)$ 
and $\mu_{\|\|}(f \mid C)$, respectively. Hence $L_{\Phi} \ni f \rightarrow \mu_{\Phi}(f \mid C) \in L_{\Phi}$ and $L_{\Phi} \ni f \rightarrow$ $\mu_{\|\|}(f \mid C) \in L_{\Phi}$ are operators on $L_{\Phi}$ and the following result states the \|\|$_{\Phi}$-continuity of these operators.

2. Corollary. Let $\Phi$ be strictly convex and assume that $L_{\Phi}=L_{\Phi}^{\infty}$. Let $C \subset L_{\Phi}$ be a $\Phi$-closed convex lattice and a cone. Then $\mu_{\Phi}(\cdot \mid C)$ and $\mu_{\|} \|(\cdot \mid C)$ are \|\|$_{\Phi}-$ continuous operators on $L_{\Phi}$.

Proof. As $L_{\Phi}=L_{\Phi}^{\infty}$ let us at first remark that

$$
\left\|h_{n}\right\|_{\Phi} \rightarrow 0 \text { iff } \int \Phi\left(a\left|h_{n}\right|\right) d \mu \rightarrow 0 \text { for all } a>0 \text {. }
$$

Let now $\left\|f_{n}-f_{0}\right\|_{\Phi} \rightarrow_{n \in N} 0$ and $N_{1} \subset \mathbf{N}$ be a subsequence. It suffices to prove that there exists a subsequence $\mathbf{N}_{2} \subset \mathbf{N}_{1}$ such that

$$
\begin{gathered}
\left\|\mu_{\Phi}\left(f_{n} \mid C\right)-\mu_{\Phi}\left(f_{0} \mid C\right)\right\|_{\Phi} \underset{n \in N_{2}}{\rightarrow} 0, \\
\left\|\mu_{\|\|}\left(f_{n} \mid C\right)-\mu_{\|\|}\left(f_{0} \mid C\right)\right\|_{\Phi} \underset{n \in N_{2}}{\vec{N}_{2}} 0 .
\end{gathered}
$$

Since $\left\|f_{n}-f_{0}\right\|_{\Phi} \rightarrow_{n \in N_{1}} 0$ there exists a subsequence $N_{2} \subset N_{1}$ such that

$$
f_{n} \underset{n \in N_{2}}{\rightarrow} f_{0} \quad \mu \text {-a.e. }
$$

and

$$
\sum_{n \in N_{2}}\left\|f_{n}-f_{0}\right\|_{\Phi}<\infty
$$

From (5) and $L_{\Phi}=L_{\Phi}^{\infty}$ we obtain

$$
\sup _{n \in N_{2}}\left|f_{n}\right|<\left|f_{0}\right|+\sum_{n \in N_{2}}\left|f_{n}-f_{0}\right| \in L_{\Phi} .
$$

Now (4), (6) and Theorem 1 imply

$$
\mu_{\Phi}\left(f_{n} \mid C\right) \underset{n \in N_{2}}{\rightarrow} \mu_{\Phi}\left(f_{0} \mid C\right) \quad \mu \text {-a.e.; } \quad \sup _{n \in N_{2}}\left|\mu_{\Phi}\left(f_{n} \mid C\right)\right| \in L_{\Phi} \text {. }
$$

Using (1), $L_{\Phi}=L_{\Phi}^{\infty}$ and the Theorem of Lebesgue, (7) implies (2). It remains to prove (3). Since $\left\|f_{n}-f_{0}\right\|_{\Phi} \rightarrow 0$ and $C$ is \|\|$_{\Phi}$-closed (see Theorem 10 of [4]) it is easy to see that

$$
\delta_{n}:=\left\|f_{n}-\mu_{\|\|}\left(f_{n} \mid C\right)\right\|_{\Phi} \underset{n \in \mathbb{N}}{\rightarrow}\left\|f_{0}-\mu_{\|\|}\left(f_{0} \mid C\right)\right\|_{\Phi}=: \delta_{0} .
$$

Let w.l.g. $\delta_{0}>0$; hence w.l.g. $\delta_{n}>0$ for all $n \in N$. According to Corollary 8 of [4] we have, as $C$ is a cone, that

$$
\mu_{\|\|}\left(f_{n} \mid C\right)=\delta_{n} \mu_{\Phi}\left(\frac{1}{\delta_{n}} f_{n} \mid C\right), \quad n \in \mathrm{N} \cup\{0\} .
$$

Since $f_{n} \rightarrow\|\|_{\odot} f_{0}$, and $\delta_{n} \rightarrow \delta_{0}$ by (8), we have

$$
\frac{1}{\delta_{n}} f_{n} \rightarrow \|_{\Phi} \frac{1}{\delta_{0}} f_{0}
$$


Hence the continuity of $\mu_{\Phi}(\cdot \mid C)$ implies

$$
\left\|\mu_{\Phi}\left(\frac{1}{\delta_{n}} f_{n} \mid C\right)-\mu_{\Phi}\left(\frac{1}{\delta_{0}} f_{0} \mid C\right)\right\|_{\Phi} \underset{n \in N}{\rightarrow} 0 .
$$

Together with (9) and (8) this yields (3).

For the special case that $C$ is the system of measurable functions with respect to a $\sigma$-field the assertion of Corollary 2 follows from Satz 5.10 of [3]. The methods used there are closely related to this special type of $C$ and cannot be transferred to arbitrary $\Phi$-closed convex lattices.

The following lemmas are the main tools for the proof of Theorem 1.

3. Lemma. Assume that $L_{\Phi}=L_{\Phi}^{\infty}$. Let $C_{i} \subset L_{\Phi}, i=1, \ldots, n$, be $\Phi$-closed lattices with $C_{1} \supset C_{2} \supset \cdots \supset C_{n}$. If $f_{i} \in L_{\Phi}$ and $g_{i} \in \mu_{\Phi}\left(f_{i} \mid C_{i}\right), i=1, \ldots, n$ then

(i) $\int \Phi\left(\left|f_{1} \wedge \cdots \wedge f_{n}-g_{1} \wedge \cdots \wedge g_{n}\right|\right) d \mu \leqslant \int \Phi\left(\left|f_{1} \wedge \cdots \wedge f_{n}-g_{n}\right|\right) d \mu$,

(ii) $\int \Phi\left(\left|f_{1} \vee \cdots \vee f_{n}-g_{1} \vee \cdots \vee g_{n}\right|\right) d \mu<\int \Phi\left(\left|f_{1} \vee \cdots \vee f_{n}-g_{n}\right|\right) d \mu$.

Proof. To show (i) it suffices to prove that for $j<n$

$$
\begin{aligned}
\int \Phi\left(\mid f_{1} \wedge \cdots\right. & \left.\wedge f_{n}-g_{j} \wedge \cdots \wedge g_{n} \mid\right) d \mu \\
& \leqslant \int \Phi\left(\left|f_{1} \wedge \cdots \wedge f_{n}-g_{j+1} \wedge \cdots \wedge g_{n}\right|\right) d \mu .
\end{aligned}
$$

As $\Phi$ is convex, Lemma 20 of [4] implies

$$
\begin{aligned}
\Phi\left(\mid f_{1} \wedge \cdots \wedge\right. & \left.f_{n}-g_{j} \wedge\left(g_{j+1} \wedge \cdots \wedge g_{n}\right) \mid\right) \\
& +\Phi\left(\left|f_{j}-g_{j} \vee\left(g_{j+1} \wedge \cdots \wedge g_{n}\right)\right|\right) \\
\leqslant & \Phi\left(\left|f_{1} \wedge \cdots \wedge f_{n}-g_{j+1} \wedge \cdots \wedge g_{n}\right|\right)+\Phi\left(\left|f_{j}-g_{j}\right|\right)
\end{aligned}
$$

Since $C_{j}$ is a lattice and $g_{i} \in C_{i} \subset C_{j}$ for $i \geqslant j$ we have $g_{j} \vee\left(g_{j+1} \wedge \cdots \wedge g_{n}\right) \in$ $C_{j}$. As $g_{j} \in \mu_{\Phi}\left(f_{j} \mid C_{j}\right)$ we obtain

$$
\int \Phi\left(\left|f_{j}-g_{j}\right|\right) d \mu \leqslant \int \Phi\left(\left|f_{j}-g_{j} \vee\left(g_{j+1} \wedge \cdots \wedge g_{n}\right)\right|\right) d \mu .
$$

Using (3) integration of (2) yields (1). This proves (i); the proof for (ii) runs by interchanging $\vee$ and $\wedge$.

4. Lemma. Assume that $L_{\Phi}=L_{\Phi}^{\infty}$. Let $0<h_{k}, r_{k} \in L_{\Phi}$ and assume that $\sup _{k \in \mathbf{N}} h_{k}$, $\sup _{k \in N} r_{k} \in L_{\Phi}$ and $r_{k} \rightarrow 0 \mu$-a.e. Then

$$
\int \Phi\left(h_{k}+r_{k}\right) d \mu-\int \Phi\left(h_{k}\right) d \mu \underset{k \in \mathbf{N}}{\rightarrow} 0
$$

Proof. Let $\Phi_{+}^{\prime}$ be the right derivative of $\Phi$. Then $\Phi_{+}^{\prime}$ is nondecreasing and $\Phi(x)=\int_{0}^{x} \Phi_{+}^{\prime}(t) d t$ (see e.g. [5]). Hence for all $k \in \mathbf{N}$

(*) $\Phi\left(h_{k}+r_{k}\right)-\Phi\left(h_{k}\right)=\int_{h_{k}}^{h_{k}+r_{k}} \Phi_{+}^{\prime}(t) d t<r_{k} \Phi_{+}^{\prime}\left(h_{k}+r_{k}\right)<r \Phi_{+}^{\prime}(h+r)$

with $r:=\sup _{k \in \mathbf{N}} r_{k} \in L_{\Phi}$ and $h:=\sup _{k \in N} h_{k} \in L_{\Phi}$. 
By the Theorem of Lebesgue (*) directly implies the assertion if we show $r \Phi_{+}^{\prime}(h+r) \in L_{1}$. As $0 \leqslant x \Phi_{+}^{\prime}(x)<\int_{x}^{2 x} \Phi_{+}^{\prime}(t) d t<\Phi(2 x)$ and $L_{\Phi}=L_{\Phi}^{\infty}$ we have $g \Phi_{+}^{\prime}(g) \in L_{1}$ if $0 \leqslant g \in L_{\Phi}$. Applying this to $g=h+r \in L_{\Phi}$ we obtain $r \Phi_{+}^{\prime}(h+r) \in L_{1}$.

\section{REFERENCES}

1. T. Ando and L. Amemiya, Almost everywhere convergence of prediction sequences in $L_{p}(1<p<$ $\infty)$, Z. Wahrsch. Verw. Gebiete 4 (1965), 113-120.

2. H. D. Brunk, Uniform inequalities for conditional p-means given o-lattices, Ann. Probab. 3 (1975), 1025-1030.

3. N. Herrndorf, Beste $\Phi$ - und $N_{\Phi}$-Approximanten in Orlicz-Räumen vektorwertiger Funktionen, Thesis, Köln, 1980.

4. D. Landers and L. Rogge, Best approximants in $L_{\Phi}$-spaces, Z. Wahrsch. Verw. Gebiete 51 (1980), 215-237.

5. W. A. J. Luxemburg and A. C. Zaanen, Riesz spaces, North-Holland, Amsterdam-London, 1971.

6. T. Shintani and T. Ando, Best approximants in $L_{1}$-space, $Z$. Wahrsch. Verw. Gebiete 33 (1975), 33-39.

Mathemattsches Institut der Universität Köln, Weyertal 86-90, D-5000 Köln 41, West Germany

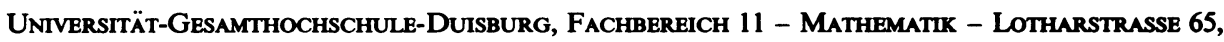
4100 Duisburg 1, West Germany 